OPEN ACCESS

Edited by:

José Bines,

National Cancer Institute (INCA), Brazil

Reviewed by:

Javier Cortes,

IOB Institute of Oncology, Spain Julio César de la Torre-Montero,

Comillas Pontifical University, Spain

*Correspondence:

Xiaosong Chen

chenxiaosong0156@hotmail.com

Kunwei Shen

kwshen@medmail.com.cn

Specialty section:

This article was submitted to

Women's Cancer,

a section of the journal

Frontiers in Oncology

Received: 31 March 2020

Accepted: 24 June 2020

Published: 31 July 2020

Citation:

Lu Y, Tong Y, Huang J, Lin L, Wu J,

Fei $X$, Huang $O$, He J, Zhu L, Chen W,

Li Y, Chen $X$ and Shen $K(2020)$

Primary 21-Gene Recurrence Score

and Disease Outcome in

Loco-Regional and Distant Recurrent Breast Cancer Patients.

Front. Oncol. 10:1315.

doi: 10.3389/fonc.2020.01315

\section{Primary 21-Gene Recurrence Score and Disease Outcome in Loco-Regional and Distant Recurrent Breast Cancer Patients}

\author{
Yujie Lu ${ }^{1}$, Yiwei Tong ${ }^{1}$, Jiahui Huang ${ }^{1}$, Lin Lin ${ }^{2}$, Jiayi Wu ${ }^{1}$, Xiaochun Fei ${ }^{3}$, Ou Huang ${ }^{1}$, \\ Jianrong He ${ }^{1}$, Li Zhu ${ }^{1}$, Weiguo Chen ${ }^{1}$, Yafen $\mathrm{Li}^{1}$, Xiaosong Chen ${ }^{1 *}$ and Kunwei Shen ${ }^{1 \star}$ \\ ${ }^{1}$ Department of General Surgery, Comprehensive Breast Health Center, Ruijin Hospital, Shanghai Jiao Tong University \\ School of Medicine, Shanghai, China, ${ }^{2}$ Department of Clinical Laboratory, Ruijin Hospital, Shanghai Jiaotong University \\ School of Medicine, Shanghai, China, ${ }^{3}$ Department of Pathology, Ruijin Hospital, Shanghai Jiao Tong University School of \\ Medicine, Shanghai, China
}

Background: The 21-gene recurrence score (RS) assay has been proven prognostic and predictive for hormone receptor-positive/HER2-negative, node-negative early breast cancer patients. However, whether primary 21-gene RS can predict prognosis in recurrent breast cancer patients remained unknown.

Patients and Methods: Consecutive breast cancer patients operated in Comprehensive Breast Health Center, Shanghai Ruijin Hospital between January 2009 and December 2018 were retrospectively analyzed. Patients with available 21 -gene RS result for the primary tumor and reporting disease recurrence during follow-up were included. Association of 21-gene RS and overall survival (OS), post-recurrence overall survival (PR-OS), post-recurrence progression-free survival (PR-PFS), and first-line systemic treatment after recurrence were compared among different groups.

Results: A total of 74 recurrent patients were included, with 10, 27, 37 patients in the RS $<18,18-30$, and $\geq 31$ groups, respectively. Recurrent patients with $\mathrm{RS} \geq 31$ were more likely to receive chemotherapy as their first-line treatment compared to those with $\mathrm{RS}<$ $31(P=0.025)$. Compared to those with $\mathrm{RS}<31$, patients with $\mathrm{RS} \geq 31$ had significantly worse OS $(P=0.025)$, worse PR-OS $(P=0.026)$, and a trend of inferior PR-PFS $(P=$ 0.106). Multivariate analysis demonstrated that primary ER expression level (OS: $P=$ 0.009; PR-OS: $P=0.017$ ) and histological grade (OS: $P=0.003$; PR-OS: $P=0.009$ ), but not primary 21-gene RS (OS: $P=0.706$; PR-OS: $P=0.120$ ), were independently associated with worse OS and PR-OS.

Conclusions: High primary 21-gene RS tended to be associated with worse disease outcome in loco-regional and distant recurrent breast cancer patients, which could influence the first-line systemic treatment after relapse, warranting further clinical evaluation.

Keywords: breast cancer, 21-gene RS, recurrence, prognosis, first-line treatment 


\section{INTRODUCTION}

Breast cancer (BC) is the most common global malignancy in women $(1,2)$. Despite standard comprehensive treatment according to clinical and histopathological features, $20-30 \%$ early-stage BC will develop loco-regional recurrence (LRR) and/or distant metastasis $(3,4)$. Previous meta-analysis involving 13,785 patients from 11 trials declared that a majority part of distant metastatic BC patients died within 2 years after recurrence (5). However, disease outcome of recurrent patients is highly variable and hard to predict, especially in hormone receptor (HR)-positive patients (6).

There are plenty of studies that analyze the predictive and prognostic factors on disease progression in early BC patients (7). Risk of BC recurrence was shown to be related to axillary lymph node status (8), primary tumor size, and tumor grade $(9,10)$. However, there remained controversies in the metastatic setting $(11,12)$. Tobin et al. revealed that the molecular subtype of metastatic lesions had prognostic value on post-recurrence survival (PR-OS) (13). Other retrospective studies demonstrated that primary ER status, adjuvant therapy, recurrence free interval, and first recurrence location were independently associated with survival in metastatic BC patients (14-16). Defining predictive and prognostic factors in the metastatic setting offered great challenges as well as opportunities toward an improved management of BC patients.

Over the past two decades, several gene expression signatures by microarray analysis have been developed in effort to predict prognosis and chemotherapy (CT) benefit (17). Among them, the 21-gene Recurrence Score (RS) assay was most widely applied and evaluated in clinical trials, which can predict both the benefit of adjuvant CT (18) and prognosis in HRpositive, human epidermal growth factor receptor-2 (HER2)negative, node-negative patients $(19,20)$. The 2019 National Comprehensive Cancer Network Clinical Practice Guideline (21) also suggested to spare selective low-risk patients from adjuvant $\mathrm{CT}$, while to apply adjuvant CT in high-risk patients based on RS results according to TAILORx and NSABP B-20 trials (22, 23). Nevertheless, the predictive or prognostic value of primary tumor 21-gene RS in recurrent BC patients has so far yet to be determined (24). Therefore, the aims of the current study are to evaluate the prognostic value of primary 21-gene RS for recurrent $\mathrm{BC}$ patients, as well as to identify whether 21-gene RS would influence subsequent first-line systemic treatment choice.

\section{PATIENTS AND METHODS}

\section{Study Population}

Consecutive $\mathrm{BC}$ patients treated in the Comprehensive Breast Health Center, Shanghai Ruijin Hospital, Shanghai Jiao Tong University School of Medicine, Shanghai, China, were retrospectively included. Eligibility criteria were as follows: (1) patients receiving surgery between January 2009 and December 2018; (2) with available 21-gene RS results on primary tumor; (3) with complete clinico-pathological characteristics and immunohistochemical results for primary tumor; (4) reporting disease recurrence during follow-up period. Detailed data were retrieved from Shanghai Jiao Tong University Breast Cancer Database (SJTU-BCDB). Current study was approved by the independent Ethical Committees of Ruijin Hospital, Shanghai Jiao Tong University School of Medicine. All procedures were in accordance with the 1964 Helsinki declaration and its later amendments.

\section{Histo-Pathological Analysis}

At least two experienced pathologists from the Department of Pathology, Ruijin Hospital, Shanghai Jiao Tong University School of Medicine, contributed to the tumor histo-pathological analysis. Positive criteria for IHC assessment of estrogen receptor (ER), progesterone receptor (PR), HER2, and Ki67 were as described in our previous reports (25). ER $\geq 50 \%$ was classified as high-expression (26). Molecular subtype was classified according to the 2013 St. Gallen expert panel consensus. Luminal A subtype was defined as ER+/PR $\geq 20 \% /$ HER2-/Ki-67 $<14 \%$, while Luminal B subtype was defined as ER+/HER2-/Ki-67 $\geq 14 \%$, or $\mathrm{ER}+/ \mathrm{PR}<20 \% / \mathrm{HER} 2-$, or ER-/PR+/HER2-.

\section{1-Gene RS Assay Testing}

The examination of the 21-gene RS assay was as reported in our previous work (23). Unstained breast tumor formalin fixed paraffin-embedded (FFPE) sections, from which RNA was extracted, were carefully selected by experienced pathologists in the Department of Pathology to ensure that tumor tissue consisted of at least $50 \%$ of the section. RNeasy FFPE RNA kit (Qiagen, 73504, Germany) and Omniscript RT kit (Qiagen, 205111, Germany) were applied in RNA extraction and reverse transcription process. Quantitative real-time polymerase chain reaction (RT-PCR) was conducted in Applied Biosystems 7500 Real-Time PCR System (Foster City, CA) using Premix Ex TaqTM (TaKaRa Bio, RR390A). The expression of genes was confirmed in triplicate, and normalized according to five reference genes Beta-actin, GAPDH, RPLPO, GUS, and TFRC. Regarding each gene expression level, patients were divided into two groups by using the median gene expression level as the cutoff value.

\section{Follow-Up}

Patient follow-up was accomplished by specialized BC nurses or staff in our center. Disease recurrence included ipsilateral and loco-regional, distant metastasis in any site, contralateral invasive $\mathrm{BC}$, and death of any cause. Overall survival (OS) was calculated from the date of surgery to death of any cause. PR-OS was computed from the date of first proven disease recurrence to death of any cause. Post-recurrence progression-free survival (PR-PFS) was estimated from the date of first proven recurrence till the date of first-line disease progression. Last follow-up was conducted in November 2019.

\section{Statistical Analysis}

Patients were classified into two risk groups: low/intermediaterisk RS ( $\mathrm{RS}<31$ ) and high-risk $\mathrm{RS}$ ( $\mathrm{RS} \geq 31$ ). Categorical data were analyzed using Chi-square test. Kaplan-Meier curve was conducted to compare OS, PR-OS, and PRPFS differences between two RS groups. Cox regression was 


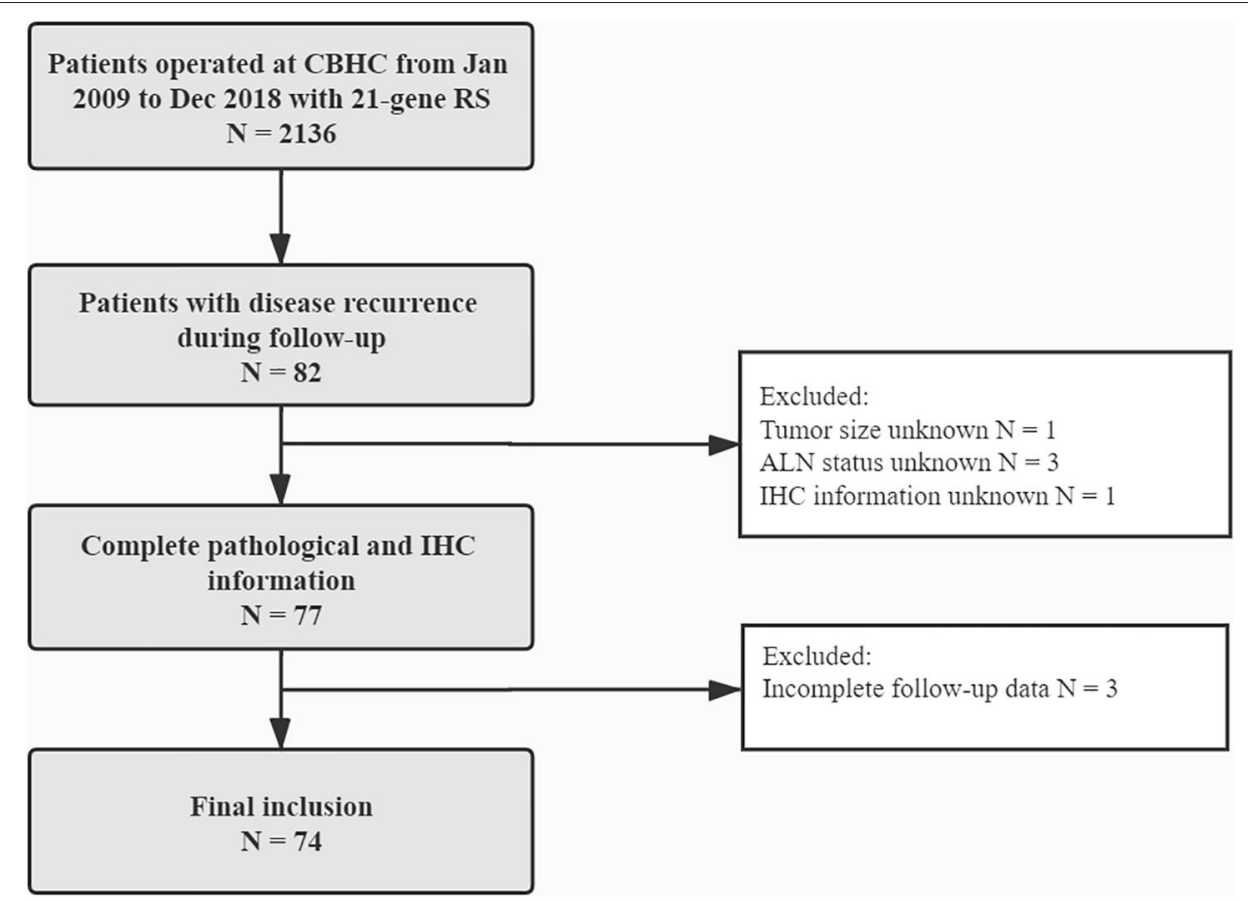

FIGURE 1 | Flowchart of included patients. CBHC, Comprehensive Breast Health Center; RS, recurrence score; ALN, axillary lymph node; IHC, immunohistochemical.

applied to identify influence factors for disease outcome. SPSS Version 23.0 (IBM Corp. Released 2015. IBM SPSS Statistics for Windows, Version 23.0. Armonk, NY: IBM Corp. from https://www.ibm.com/support/pages/how-cite-ibmspss-statistics-or-earlier-versions-spss) and GraphPad Prism version 7.0 (GraphPad Software, CA, USA) were used in the analysis and data interpretation. Two-sided $P<0.05$ was considered statistically significant.

\section{RESULTS}

\section{Patient Baseline Characteristics}

In all, 2,136 BC patients with 21-gene RS records were retrospectively reviewed, and their characteristics were listed in Supplementary Table S1. Eighty-two patients reported disease recurrence during follow-up, including $20 \mathrm{LRR}$ and 62 distant metastases. Patients with incomplete pathological, or followup information were then excluded. Overall, 74 patients were enrolled for final analysis (Figure 1). Nineteen patients (Table 2) reported LRR alone, 55 patients had distant metastasis with or without LRR.

Detailed clinico-pathological features were presented in Table 1. Median age was 53.0 (range 24-84) years. Fortyfive patients were post-menopausal. Regarding primary surgery, mastectomy was performed in 44 patients and 37 patients received axillary lymph node dissection. Thirty-six patients had tumor larger than $2.0 \mathrm{~cm}$, while $79.73 \%$ patients $(N=59)$ were node-negative. Sixty patients were diagnosed as invasive ductal carcinoma and grade 3 tumors were observed in 15 patients. Fifty-eight patients had ER $\geq 50 \%$. Thirty-seven patients had PR
$<20 \%$, of whom 17 were PR-negative. Luminal-B-like subtypes accounted for $87.84 \%(N=65)$ of the whole study population. According to 21-gene RS, 10 (12.99\%), 27 (37.66\%), and 37 $(49.35 \%)$ patients were categorized into low, intermediate, and high-risk groups, respectively.

\section{Association of 21-Gene RS and First-Line Treatment Recommendation}

Figure 2 shows the first-line treatment selections after disease recurrence in patients with different RS. Therapy information was not available for 5 patients who received first-line treatments in their local centers. There were $20.29 \%(N=14), 49.28 \%$ $(N=34)$, and $17.39 \%(N=12)$ of enrolled patients received CT, endocrine therapy (ET), and CT followed by ET (CT-ET) as their first-line treatment, respectively. In addition, 6 patients with LRR alone or oligometastatic lesion underwent surgical resection without further systemic treatment. The rest 3 patients didn't receive further treatment due to elder age or other socioeconomic factors.

As first-line systemic treatment in recurrent BC patients, CT, CT-ET, and ET were assigned to 10 (29.41\%), 8 (23.53\%), and $13(38.24 \%)$ patients with $\mathrm{RS} \geq 31$, and $4(11.43 \%), 4$ $(11.43 \%)$, and $21(60.00 \%)$ patients with $\mathrm{RS}<31$, respectively. Six (17.14\%) low/intermediate-risk patients were spared from systemic treatment after recurrence. Compared to patients with primary $\mathrm{RS}<31$, patients with primary $\mathrm{RS} \geq 31$ were more likely to receive CT as first-line treatment $(P=0.013)$.

When we adopted RS $\geq 26$ as cutoff value to identify low/intermediate or high risk, details of firstline systemic treatment after relapse were described in 
TABLE 1 | Baseline clinico-pathological characteristics of enrolled population.

\begin{tabular}{|c|c|c|}
\hline & $N$ & $\%$ \\
\hline Age, years (median, range) & $53.0(24-84)$ & \\
\hline$\leq 50$ years & 27 & 36.49 \\
\hline$>50$ years & 47 & 63.51 \\
\hline \multicolumn{3}{|l|}{ Menstrual status } \\
\hline Pre-menopausal & 29 & 39.19 \\
\hline Post-menopausal & 45 & 60.81 \\
\hline \multicolumn{3}{|l|}{ Breast surgery } \\
\hline Mastectomy & 44 & 59.46 \\
\hline BCS & 30 & 40.54 \\
\hline \multicolumn{3}{|l|}{ Axillary surgery } \\
\hline SLNB & 37 & 50.00 \\
\hline ALND & 37 & 50.00 \\
\hline \multicolumn{3}{|l|}{ Histological type } \\
\hline IDC & 60 & 81.08 \\
\hline Others & 14 & 18.92 \\
\hline \multicolumn{3}{|l|}{ Histological grade } \\
\hline 2 & 45 & 60.81 \\
\hline 3 & 15 & 20.27 \\
\hline NA & 14 & 18.92 \\
\hline \multicolumn{3}{|l|}{ Tumor size } \\
\hline$\leq 2 \mathrm{~cm}$ & 38 & 51.35 \\
\hline$>2 \mathrm{~cm}$ & 36 & 48.65 \\
\hline \multicolumn{3}{|l|}{ ALN involvement } \\
\hline Negative & 59 & 79.73 \\
\hline Positive & 15 & 20.27 \\
\hline \multicolumn{3}{|l|}{ ER } \\
\hline$<50 \%$ & 16 & 21.62 \\
\hline$\geq 50 \%$ & 58 & 78.38 \\
\hline \multicolumn{3}{|l|}{ PR } \\
\hline$<20 \%$ & 37 & 50.00 \\
\hline$\geq 20 \%$ & 37 & 50.00 \\
\hline \multicolumn{3}{|l|}{ Ki67 } \\
\hline$<14 \%$ & 24 & 32.43 \\
\hline$\geq 14 \%$ & 50 & 67.57 \\
\hline \multicolumn{3}{|l|}{ Molecular subtype } \\
\hline Luminal A & 9 & 12.16 \\
\hline Luminal B & 65 & 87.84 \\
\hline \multicolumn{3}{|l|}{ 21-gene RS } \\
\hline$<18$ & 10 & 13.51 \\
\hline $18-30$ & 27 & 36.49 \\
\hline$\geq 31$ & 37 & 50.00 \\
\hline
\end{tabular}

BCS, breast conserving surgery; SLNB, sentinel lymph node biopsy; ALND, axillary lymph node dissection; IDC, invasive ductal carcinoma; NA, not available; ALN, axillary lymph node; $E R$, estrogen receptor; $P R$, progesterone receptor; $R S$, recurrence score.

Supplementary Figure S1. CT, CT-ET, and ET were assigned to 11 (24.44\%), 9 (20.00\%), and, 21 (46.67\%) patients with primary RS $\geq 26$, and 3 (12.50\%), 3 (12.50\%) and, 13 (54.17\%) patients with $\mathrm{RS}<26$, respectively. There was no statistically difference in first-line CT choice between primary $\mathrm{RS} \geq 26$ and $\mathrm{RS}<26$ patients after recurrence $(P=0.127)$.

\section{Association of 21-Gene RS and Disease Outcome in Recurrent BC Patients}

Overall, during the follow-up of 2,136 patients with 21-gene RS, 82 patients had disease recurrence, 29 patients developed second primary malignancy, and 28 died (Supplementary Table S1). Among 74 patients finally included in our study with disease recurrence, the median follow-up was 57.90 months (range 7.60121.50) and median post-recurrence follow-up was 25.70 months (range 0.37-89.47). Fifteen patients (Table 2) experienced disease progression after recurrence, in which 10 patients died. In patients with primary RS $\geq 31,11$ patients developed disease progression and 9 patients died. The rates of 5-year OS, 2year PR-OS, and 2-year PR-PFS were 85.2, 72.5, and 66.7\%, respectively. In patients with primary RS $<31,4$ patients progressed and 1 patient died. The 5-year OS, 2-year PR-OS, and 2-year PR-PFS were 91.3, 81.6, and 90.1\%, respectively. Patients with RS $\geq 31$ had a significantly worse $\operatorname{OS}(P=0.025$, Figure $3 \mathrm{~A})$ and PR-OS $(P=0.026$, Figure 3B) compared to patients with RS $<31$. In addition, histological grade (OS: $P<0.001$; PROS: $P=0.003$, Table 3) and ER expression level (OS: $P=$ 0.026; PR-OS: $P=0.008$ ) were also related to OS and PR-OS in univariate model. PR expression level $(P=0.040)$, not RS categories $(P=0.106$, Figure $3 \mathrm{C})$, was associated with PR-PFS in univariate analysis.

In multivariate Cox regression, primary ER expression level and histological grade independently influenced OS (Supplementary Table S2) and PR-OS (Supplementary Table S3). However, 21-gene RS categories was no longer an independent impact factor for OS $(P=0.706)$ or PR-OS $(P=0.120)$ after adjusting above factors. Primary ER high-expression was associated with better OS (hazard ratio $[\mathrm{HR}]=0.11,95 \%$ confidence interval $[\mathrm{CI}] 0.02-0.57, P=$ $0.009)$ and PR-OS (HR $=0.18,95 \%$ CI $0.05-0.74, P=0.017)$ in recurrent $\mathrm{BC}$ patients. Patients with histological grade 3 tumor had a significantly worse OS $(\mathrm{HR}=4.20,95 \% \mathrm{CI} 1.65-10.65, P=$ 0.003 ) and PR-OS (HR $=7.08,95 \% \mathrm{CI} 1.64-30.63, P=0.009$ ).

When further stratified by recurrence site, LRR patients had similar clinical outcomes (OS: $P=0.439$; PR-OS: $P=0.439$; PRPFS: $P=0.695)$ with different RS. Meantime, distant metastatic patients with RS $\geq 31$ had a statistically significant worse OS ( $P$ $=0.031$, Figure 3D $),$ PR-OS $(P=0.026$, Figure 3E $)$ and PR-PFS $(P=0.030$, Figure 3F) compared to those with RS $<31$.

In addition, if RS $\geq 26$ was applied as cutoff, we observed that primary RS $\geq 26$ was associated with a worse OS in univariate analysis $(P=0.048$, Supplementary Figure $2 \mathrm{~A})$, but not with PR-OS $(P=0.182)$ or PR-PFS $(P=0.333)$. Primary RS $\geq 26$ was no longer associated with OS in multivariate analysis $(P=0.552$, Supplementary Table S4).

\section{Association of Single Gene Expression in 21-Gene RS and Disease Outcome in Recurrent BC Patients}

Regarding each gene expression and survival, univariate analysis found that $P R(P=0.007$, Table 4), CD68 $(P=0.004)$, and GSTM1 $(P=0.004)$ expression levels were significantly associated with OS. GRB7 $(P=0.046), P R(P=0.001), C D 68$ 


\begin{tabular}{|c|c|c|c|c|c|c|}
\hline \multirow[b]{2}{*}{ All patients- } & CT & C & CT-ET & ET & - & Non \\
\hline & $20.29 \%$ & \multicolumn{2}{|l|}{$17.39 \%$} & \multicolumn{2}{|l|}{$49.28 \%$} & $13.04 \%$ \\
\hline$R S \geq 31-$ & $29.41 \%$ & \multicolumn{2}{|r|}{$23.53 \%$} & \multicolumn{2}{|c|}{$38.24 \%$} & $8.82 \%$ \\
\hline \multirow[t]{3}{*}{$\mathbf{R S}<31-$} & $11.43 \% \quad 11.43 \%$ & \multicolumn{3}{|c|}{$60.00 \%$} & \multicolumn{2}{|r|}{$17.14 \%$} \\
\hline & $20 \%$ & & $40 \%$ & $60 \%$ & $80 \%$ & $100 \%$ \\
\hline & CT & & CT-ET & ET & & Non \\
\hline All patients & 14 & & 12 & 34 & & 9 \\
\hline $\mathrm{RS} \geq 31$ & 10 & & 8 & 13 & & 3 \\
\hline$R S<31$ & 4 & & 4 & 21 & & 6 \\
\hline
\end{tabular}

FIGURE 2 | Distribution of first line systemic treatment recommendation after disease recurrence. RS, recurrence score; CT, chemotherapy; ET, endocrine therapy.

TABLE 2 | Disease recurrence and survival profile of enrolled patients.

\begin{tabular}{llll}
\hline & All patients & $\mathbf{R S ~}<\mathbf{3 1}$ & $\mathbf{R S} \geq \mathbf{3 1}$ \\
\hline $\boldsymbol{N}$ & 74 & 37 & 37 \\
Progression after recurrence & 15 & 4 & 11 \\
Death after recurrence & 10 & 1 & 9 \\
Recurrence sites & & & \\
Loco-regional recurrence & 19 & 7 & 12 \\
Distant metastasis & 55 & 32 & 25 \\
$\quad$ Visceral & 21 & 13 & 8 \\
Bone or soft tissues & 30 & 17 & 13 \\
CNS & 4 & 0 & 4
\end{tabular}

$R S$, recurrence score; OS, overall survival; PR-OS, post-recurrence survival; PR-PFS, post-recurrence progression free survival; CNS, central nervous system.

$(P=0.003)$, and GSTM1 $(P=0.001)$ expression levels were significantly associated with PR-OS. GSTM1 $(P=0.040)$ was significantly associated with PR-PFS. After adjusting clinicopathological factors, gene expression level of CD68 maintained independent association to OS (High vs. low-expression, $\mathrm{HR}=$ $0.07,95 \%$ CI $0.01-0.68, P=0.022$ ) and PR-OS (High vs. lowexpression, $\mathrm{HR}=0.09,95 \% \mathrm{CI} 0.01-0.79, P=0.029)$ in recurrent BC patients.

\section{DISCUSSION}

In our current study, we included 74 patients from 2,136 consecutive patients with recurrent disease and found that patients with $\mathrm{RS} \geq 31$ had significantly worse OS and PROS and a trend of inferior PR-PFS compared with those with $\mathrm{RS}<31$. In addition, recurrent patients with $\mathrm{RS} \geq 31$ were more likely to receive CT as their first-line systemic treatment compared to those with RS $<31$. Moreover, we found several genes in RS assay were associated with disease outcome and CD68 was independently associated with OS and PR-OS in recurrent BC patients.

$\mathrm{BC}$ recurrence and metastasis are the very major reasons for poor prognosis, causing almost half of BC death within 5 year (27-29). Unlike in early BC, the widely-accepted impact factors on cancer patients' outcome, such as tumor size and lymph node status, showed no influence on survival in metastatic setting (7), which were confirmed in our study. The 21-gene $\mathrm{RS}$ is the most widely used multigene assay in early BC with predictive and prognostic value in $\mathrm{HR}+/ \mathrm{HER} 2-$, node-negative patients $(19,22)$, and it showed independently prognostic value for survival in ER+/HER2- de novo stage IV BC patients in a previous prospective study (11). However, its prognostic or predictive value in recurrent BC patients is still undetermined. Falato et al. estimated 21-gene RS and other multigene signatures in 187 recurrent BC patients using cDNA Affymetrix GPL10379 microarray data (24). They did not find any association between primary 21-gene RS and patient's survival in metastatic setting. While in our study, we applied qRT-PCR method to determine the gene expression and calculated RS, and found that primary high-risk RS patients had a significant worse OS and PR-OS in the whole population, and an inferior OS, PR-OS, and PRPFS in patients with distant metastatic disease. Such different findings between studies may be attributed to different study 

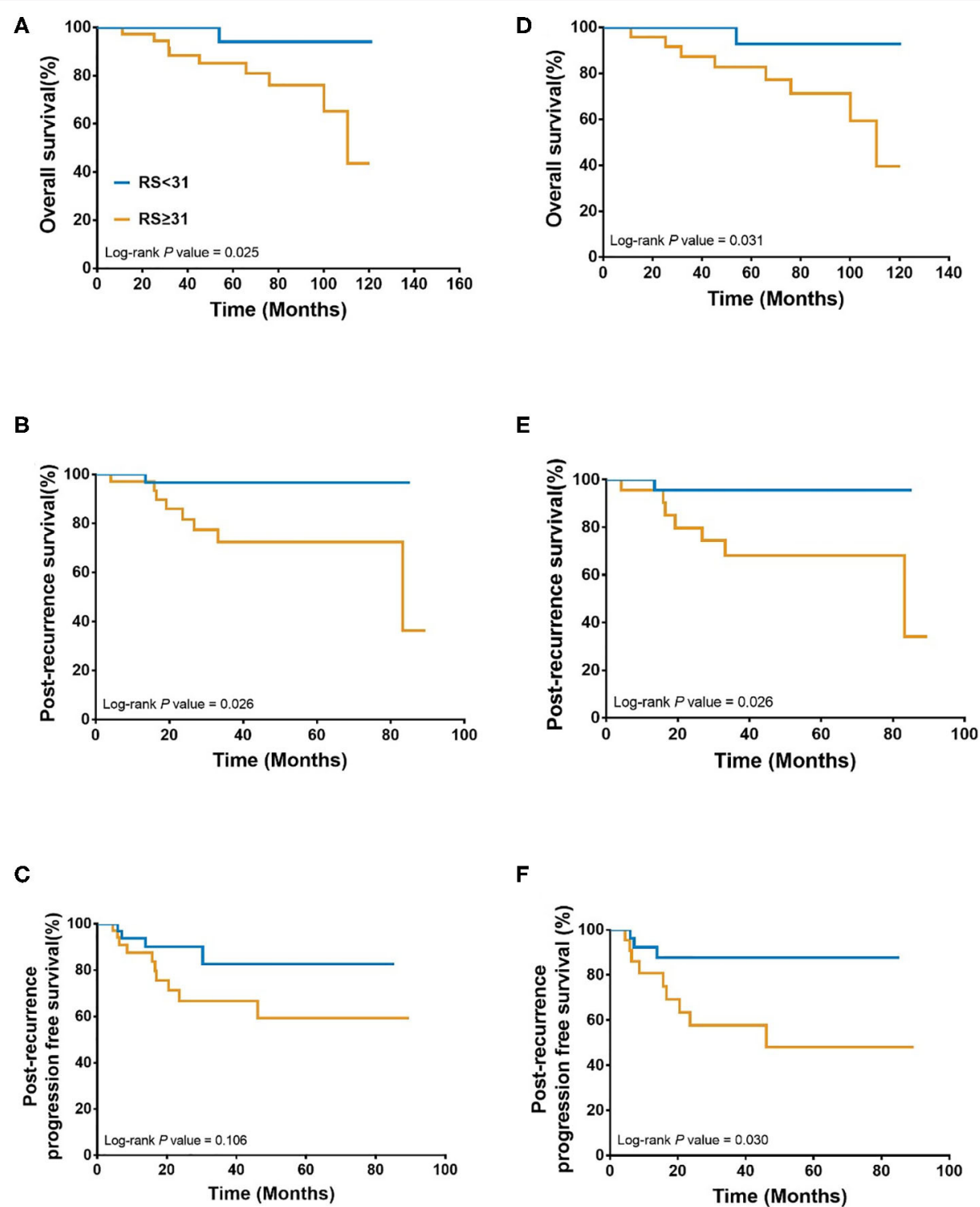

FIGURE 3 | Association between 21-gene RS and survival in recurrent breast cancer patients. Overall survival (A), Post recurrence survival (B), and Post recurrence progression free survival (C) in the whole cohort; Overall survival (D), Post recurrence survival (E), and Post recurrence progression free survival (F) in patients with distant metastasis patients. RS, recurrence score.

population and different testing techniques. Moreover, Falato's study included HR- or HER2 + BC patients, which was not indication for 21-gene RS testing right now (30). Furthermore, we found that primary ER low-expression and histological grade 3 tumor were associated with worse OS and PR-OS in recurrent patients, which was in consistent with previous studies and may guide our further patient risk classification and treatment decision $(29,31,32)$.

Systemic treatment decision for recurrent BC patients was largely based on biomarker status, number and sites of recurrence, and response of prior therapies (21, 33). For $\mathrm{ER}+/ \mathrm{HER} 2$ - recurrent $\mathrm{BC}$ patients, ET is the preferred option, whereas CT is mainly assigned to patients with visceral crisis (21). The 21-gene RS is widely used to guide adjuvant treatment decision in early BC patients, however, its value in metastatic BC patients was limited. King et al. evaluated that in ER+/HER2de novo stage IV BC patients, CT was more likely to be assigned to intermediate/high-risk RS patients as first-line systemic treatment and more with low-risk RS patients received firstline ET (11). Currently, there was no consensus on the optimal cutoff value applicable in metastatic setting. Here in this study we adopted both 31 and 26 as cutoffs, and found that primary 21gene $R S \geq 31$, but not $R S \geq 26$ was associated with significantly increased CT usage after recurrence in ER+/HER2- patients. 
TABLE 3 | Univariate analysis of impact factors on OS, PR-OS, and PR-PFS.

\begin{tabular}{lccc}
\hline Clinico-pathological characteristics & \multicolumn{3}{c}{ P-value } \\
\cline { 2 - 4 } & OS & PR-OS & PR-PFS \\
\hline Age & 0.390 & 0.395 & 0.191 \\
Menstrual status & 0.627 & 0.566 & 0.905 \\
Histological type & 0.099 & 0.106 & 0.061 \\
Histological grade & $<\mathbf{0 . 0 0 1}$ & $\mathbf{0 . 0 0 3}$ & 0.262 \\
Tumor size & 0.912 & 0.978 & 0.818 \\
ALN involvement & 0.708 & 0.592 & 0.636 \\
ER & $\mathbf{0 . 0 2 6}$ & $\mathbf{0 . 0 0 8}$ & 0.273 \\
PR & 0.114 & 0.055 & $\mathbf{0 . 0 4 0}$ \\
Ki67 & 0.219 & 0.159 & 0.673 \\
RS & $\mathbf{0 . 0 2 5}$ & $\mathbf{0 . 0 2 6}$ & 0.106
\end{tabular}

OS, overall survival; PR-OS, post-recurrence survival; PR-PFS, post-recurrence progression free survival; $A L N$, axillary lymph node; $E R$, estrogen receptor; $P R$, progesterone receptor; $R S$, recurrence score. Bold values are statistically significant.

TABLE 4 | Univariate analysis of association between single gene expression in primary tumor and OS, PR-OS, and PR-PFS.

\begin{tabular}{lccc}
\hline Gene expression & \multicolumn{3}{c}{ P-value } \\
\cline { 2 - 4 } & OS & PR-OS & PR-PFS \\
\hline GRB7 & 0.122 & 0.046 & 0.648 \\
HER2 & 0.100 & 0.109 & 0.998 \\
ER & 0.050 & 0.051 & 0.128 \\
PR & $\mathbf{0 . 0 0 7}$ & $\mathbf{0 . 0 0 4}$ & 0.086 \\
BCI2 & 0.208 & 0.353 & 0.244 \\
CEGP & 0.309 & 0.217 & 0.332 \\
CCNB1 & 0.415 & 0.206 & 0.735 \\
Ki67 & 0.765 & 0.966 & 0.610 \\
MYBL2 & 0.188 & 0.257 & 0.519 \\
STK15 & 0.930 & 0.675 & 0.493 \\
SURV & 0.192 & 0.107 & 0.769 \\
CTSL2 & 0.510 & 0.176 & 0.962 \\
STMY3 & 0.320 & 0.630 & 0.336 \\
CD68 & $\mathbf{0 . 0 0 4}$ & $\mathbf{0 . 0 0 3}$ & 0.075 \\
GSTM1 & $\mathbf{0 . 0 0 4}$ & $\mathbf{0 . 0 0 1}$ & $\mathbf{0 . 0 4 0}$ \\
BAG1 & 0.553 & 0.377 & 0.554 \\
\hline
\end{tabular}

OS, overall survival; PR-OS, post-recurrence survival; PR-PFS, post-recurrence progression free survival. Bold values are statistically significant.

Herein, our finding suggested that primary 21-gene RS could influence first-line systemic treatment choice in the recurrent setting, and $\mathrm{RS} \geq 31$ might be the preferred cutoff for clinicians to decide first-line systemic treatment after disease recurrence, which warranted further validation. In addition, Asad et al. found that RS showed different predictive value of CT benefit in patients with different primary tumor size in adjuvant setting (34). In our study cohort, 6,31 , and 32 patients had a primary tumor of $\leq 1$, $1-2$, and $>2 \mathrm{~cm}$. 21-gene RS could predict chemotherapy choice in $1-2 \mathrm{~cm}$ tumors $(P=0.032$, Supplementary Table S5), but not in tumor $\leq 1 \mathrm{~cm}(P=1.000)$, or $>2 \mathrm{~cm}(P=0.433)$, suggesting that primary tumor size may influence predictive value of 21 gene RS on CT choice in metastatic setting. However, the sample size of each subgroup was relatively small, which warrants further exploration with larger cohort and longer follow-up time.

Previous studies have demonstrated that single gene expression in 21-gene RS panel was associated with survival in early BC patients. GSTM1 $(35,36), E R$, and $P R$ (37) were associated with better outcome, while CD68 (38) and GRB7 (39) was related with worse prognosis. In our study, we found that $P R$ and GSTM1 was independently associated with superior disease outcome. However, in contrast, our study found that patients with high expression level of GRB7 or CD68 had a better disease outcome. The possible reasons were our study only included patients who had disease recurrence. In addition, molecular biomarkers and gene expression would change after disease recurrence (40-42). Two earlier prospective studies demonstrated that ER, PR, and HER2 status changed between primary and recurrent lesions in $12.6,31.2$, and $5.5 \%$ of patients, respectively $(43,44)$. Moreover, 21 -gene RS retest on recurrent $\mathrm{BC}$ lesions is still doubtful, and we didn't know the true concordance rate of 21-gene RS between primary and recurrent $\mathrm{BC}$, deserving further clinical evaluation.

In current study, we firstly evaluate the prognostic value of primary 21-gene RS categories on recurrent BC patients and its influence on first-line systemic treatment decision after recurrence. Several limitations still existed in this study. Firstly, as a retrospective analysis, selection bias in study population was unavoidable. Besides, the diagnosis of disease recurrence was based on radiographic examination or histo-pathological result. So only patients with "observable" or "evaluable" event could be included, which might lead to deviation in time and diagnosis of recurrence. In addition, it was difficult to distinguish a true ipsilateral local recurrence developed after breast conservation with a new primary tumor, which may result in potential bias. In our study cohort, 6 patients developed new tumors in the ipsilateral breast, and all of their new tumors occurred in the same quadrant of primary tumor, had similar histology to primary tumor, and thus were considered as disease recurrence. Further molecular analysis of clonal differences should be accomplished to better classify the patients (45). What's more, the sample size of the study was relatively small to conduct additional subgroup analysis. Last but not least, median post-recurrence follow-up time was relatively short to explore the subsequent systemic treatment and its influence on PR-OS.

In conclusion, our study demonstrated that primary 21-gene RS tended to be associated with worse disease outcome in locoregional recurrent and distant metastatic patients. Several genes in 21-gene RS panel showed prognostic value for recurrent $\mathrm{BC}$, which requires further evaluation. Primary 21-gene RS could influence the first-line systemic treatment after relapse, warranting clinical validation.

\section{DATA AVAILABILITY STATEMENT}

The raw data supporting the conclusions of this article will be made available by the authors, without undue reservation. 


\section{ETHICS STATEMENT}

The study involving human participants was reviewed and approved by the independent Ethical Committees of Ruijin Hospital, Shanghai Jiao Tong University School of Medicine. The patients/participants provided their written informed consent to participate in this study.

\section{AUTHOR CONTRIBUTIONS}

All authors listed have made a substantial, direct and intellectual contribution to the work, and approved it for publication.

\section{FUNDING}

The authors received financial supported from the National Natural Science Foundation of China (Grant Number: 81772797), Shanghai Municipal Education Commission Gaofeng Clinical Medicine Grant Support (20172007); Ruijin
Hospital, Shanghai Jiao Tong University School of MedicineGuangci Excellent Youth Training Program (GCQN-2017-A18). All these financial sponsors had no role in the study design, information collection, data analysis or interpretation.

\section{ACKNOWLEDGMENTS}

The authors would like to thank the assistance of Ms. Yidong Du in inputting SJTU-BCDB.

\section{SUPPLEMENTARY MATERIAL}

The Supplementary Material for this article can be found online at: https://www.frontiersin.org/articles/10.3389/fonc. 2020.01315/full\#supplementary-material

Supplementary Figure $\mathbf{S 1}$ | Distribution of first line systemic treatment recommendation after disease recurrence when adopted $\mathrm{RS} \geq 26$ as cutoff value.

Supplementary Figure S2 | Association between 21-gene RS and survival in recurrent breast cancer patients when adopted $R S \geq 26$ as cutoff value. Overall survival (A), Post recurrence survival (B), and Post recurrence progression free survival (C) in the whole.

\section{REFERENCES}

1. Bray F, Ferlay J, Soerjomataram I, Siegel RL, Torre LA, Jemal A. Global cancer statistics 2018: GLOBOCAN estimates of incidence and mortality worldwide for 36 cancers in 185 countries. CA Cancer J Clin. (2018) 68:394424. doi: $10.3322 / \mathrm{caac} .21492$

2. Siegel RL, Miller KD, Jemal A. Cancer statistics 2020. CA Cancer J Clin. (2020) 70:7-30. doi: 10.3322/caac. 21590

3. Early Breast Cancer Trialists' Collaborative Group (EBCTCG). Effects of chemotherapy and hormonal therapy for early breast cancer on recurrence and 15-year survival: an overview of the randomised trials. Lancet. (2005) 365:1687-717. doi: 10.1016/S0140-6736(05)66544-0

4. Perez EA, Romond EH, Suman VJ, Jeong JH, Davidson NE, Geyer CE, et al. Four-year follow-up of trastuzumab plus adjuvant chemotherapy for operable human epidermal growth factor receptor 2-positive breast cancer: joint analysis of data from NCCTG N9831 and NSABP B-31. J Clin Oncol. (2011) 29:3366-73. doi: 10.1200/JCO.2011.35.0868

5. Tevaarwerk AJ, Gray RJ, Schneider BP, Smith ML, Wagner LI, Fetting JH, et al. Survival in patients with metastatic recurrent breast cancer after adjuvant chemotherapy: little evidence of improvement over the past 30 years. Cancer. (2013) 119:1140-8. doi: 10.1002/cncr.27819

6. Narod SA, Giannakeas V, Sopik V. Time to death in breast cancer patients as an indicator of treatment response. Breast Cancer Res Treat. (2018) 172:65969. doi: 10.1007/s10549-018-4935-3

7. Sopik V, Sun P, Narod SA. Predictors of time to death after distant recurrence in breast cancer patients. Breast Cancer Res Treat. (2019) 173:46574. doi: 10.1007/s10549-018-5002-9

8. Ahn SG, Lee HM, Cho SH, Bae SJ, Lee SA, Hwang SH, et al. The difference in prognostic factors between early recurrence and late recurrence in estrogen receptor-positive breast cancer: nodal stage differently impacts early and late recurrence. PLoS ONE. (2013) 8:e63510. doi: 10.1371/journal.pone.00 63510

9. Stuart-Harris R, Dahlstrom JE, Gupta R, Zhang Y, Craft P, Shadbolt B. Recurrence in early breast cancer: analysis of data from 3,765 Australian women treated between 1997 and 2015. Breast. (2019) 44:1539. doi: 10.1016/j.breast.2019.02.004

10. Pan H, Gray R, Braybrooke J, Davies C, Taylor C, McGale P, et al. 20year risks of breast-cancer recurrence after stopping endocrine therapy at 5 years. N Engl J Med. (2017) 377:1836-46. doi: 10.1056/NEJMoa17 01830
11. King TA, Lyman JP, Gonen M, Voci A, De Brot M, Boafo C, et al. Prognostic impact of 21-gene recurrence score in patients with stage IV breast cancer: TBCRC 013. J Clin Oncol. (2016) 34:2359-65. doi: 10.1200/JCO.2015.63.1960

12. Delpech Y, Wu Y, Hess KR, Hsu L, Ayers M, Natowicz R., et al Ki67 expression in the primary tumor predicts for clinical benefit and time to progression on first-line endocrine therapy in estrogen receptorpositive metastatic breast cancer. Breast Cancer Res Treat. (2012) 135:61927. doi: 10.1007/s10549-012-2194-2

13. Tobin NP, Lindström LS, Carlson JW, Bjöhle J, Bergh J, Wennmalm K. Multi-level gene expression signatures, but not binary, outperform Ki67 for the long term prognostication of breast cancer patients. Mol Oncol. (2014) 8:741-52. doi: 10.1016/j.molonc.2014.02.007

14. Foukakis T, Fornander T, Lekberg T, Hellborg H, Adolfsson J, Bergh J. Agespecific trends of survival in metastatic breast cancer: 26 years longitudinal data from a population-based cancer registry in Stockholm, Sweden. Breast Cancer Res Treat. (2011) 130:553-60. doi: 10.1007/s10549-011-1594-Z

15. Rogoz B, de l'Aulnoit AH, Duhamel A, de l'Aulnoit DH. Thirty-year trends of survival and time-varying effects of prognostic factors in patients with metastatic breast cancer-a single institution experience. Clin Breast Cancer. (2018) 18:246-53. doi: 10.1016/j.clbc.2017.08.012

16. Arciero CA, Guo Y, Jiang R, Behera M, O’Regan R, Peng L, et al. $\mathrm{ER}(+) / \mathrm{HER} 2(+)$ breast cancer has different metastatic patterns and better survival than ER(-)/HER2(+) breast cancer. Clin Breast Cancer. (2019) 19:236-45. doi: 10.1016/j.clbc.2019.02.001

17. Geffen DB. Should decisions on adding adjuvant chemotherapy in early-stage ER-positive breast cancer be based on gene expression testing or clinicopathologic factors or both? Ann Oncol. (2018) 29:1096-8. doi: 10.1093/annonc/mdy115

18. Sparano JA, Gray RJ, Ravdin PM, Makower DF, Pritchard KI, Albain KS, et al. Clinical and genomic risk to guide the use of adjuvant therapy for breast cancer. N Engl J Med. (2019) 380:2395-405. doi: 10.1056/NEJMoa1904819

19. Sparano JA, Gray RJ, Makower DF, Pritchard KI, Albain KS, Hayes DF, et al. Adjuvant chemotherapy guided by a 21-Gene expression assay in breast cancer. N Engl J Med. (2018) 379:111-21. doi: 10.1056/NEJMoa1804710

20. Tong Y, Wu J, Huang O, He J, Zhu L, Chen W, et al. 21-gene recurrence score and adjuvant chemotherapy decision for breast cancer patients with positive lymph nodes. Sci Rep. (2019) 9:13123. doi: 10.1038/s41598-019-49644-6

21. Gradishar W. NCCN Clinical Practice Guidelines in Oncology: Breast Cancer. Version 3.2019 (2019). To view the most recent version of these guidelines, visit NCCN.org (accessed September 9, 2019). 
22. Paik S, Tang G, Shak S, Kim C, Baker J, Kim W, et al. Gene expression and benefit of chemotherapy in women with node-negative, estrogen receptor-positive breast cancer. J Clin Oncol. (2006) 24:372634. doi: 10.1200/JCO.2005.04.7985

23. Wu J, Fang Y, Lin L, Fei X, Gao W, Zhu S, et al. Distribution patterns of 21-gene recurrence score in 980 Chinese estrogen receptor-positive, HER2-negative early breast cancer patients. Oncotarget. (2017) 8:3870616. doi: 10.18632/oncotarget.16313

24. Falato C, Tobin NP, Lorent J, Lindström LS, Bergh J, Foukakis $\mathrm{T}$. Intrinsic subtypes and genomic signatures of primary breast cancer and prognosis after systemic relapse. Mol Oncol. (2016) 10:517-25. doi: 10.1016/j.molonc.2015.11.004

25. Zhu S, Wu J, Huang O, He J, Zhu L, Li Y, et al. Clinicopathological features and disease outcome in breast cancer patients with hormonal receptor discordance between core needle biopsy and following surgical sample. Ann Surg Oncol. (2019) 26:2779-86. doi: 10.1245/s10434-019-07480-y

26. Goldhirsch A, Ingle JN, Gelber RD, Coates AS, Thurlimann B, Senn HJ, et al. Thresholds for therapies: highlights of the St Gallen International expert consensus on the primary therapy of early breast cancer 2009. Ann Oncol. (2009) 20:1319-29. doi: 10.1093/annonc/mdp322

27. Lee ES, Jung SY, Kim JY, Kim JJ, Yoo TK, Kim YG, et al. Identifying the potential long-term survivors among breast cancer patients with distant metastasis. Ann Oncol. (2016) 27:828-33. doi: 10.1093/annonc/mdw036

28. Di Meglio A, Freedman RA, Lin NU, Barry WT, Metzger-Filho O, Keating NL, et al. Time trends in incidence rates and survival of newly diagnosed stage IV breast cancer by tumor histology: a population-based analysis. Breast Cancer Res Treat. (2016) 157:587-96. doi: 10.1007/s10549-016-3845-5

29. Xiong Z, Deng G, Huang X, Li X, Xie X, Wang J, et al. Score for the survival probability in metastasis breast cancer: a nomogram-based risk assessment model. Cancer Res Treat. (2018) 50:1260-9. doi: 10.4143/crt.2017.443

30. Hong J, Chen XS, Wu JY, Huang O, Zhu L, He JR, et al. [Analysis of the factors influencing adjuvant chemotherapy decisions for triple negative breast cancer]. Zhonghua zhong liu za zhi. (2017) 39:3943. doi: 10.3760/cma.j.issn.0253-3766.2017.01.008

31. Largillier R, Ferrero JM, Doyen J, Barriere J, Namer M, Mari V, et al. Prognostic factors in 1,038 women with metastatic breast cancer. Ann Oncol. (2008) 19:2012-9. doi: 10.1093/annonc/mdn424

32. Kim HJ, Ahn SG, Lee HM, Park JT, Han K, Lee SA, et al. Metastasisfree interval is closely related to tumor characteristics and has prognostic value in breast cancer patients with distant relapse. J Breast Cancer. (2015) 18:371-7. doi: 10.4048/jbc.2015.18.4.371

33. Cardoso F, Senkus E, Costa A, Papadopoulos E, Aapro M, Andre F, et al. 4th ESO-ESMO International consensus guidelines for advanced breast cancer (ABC 4)dagger. Ann Oncol. (2018) 29:1634-57. doi: 10.1093/annonc/mdy192

34. Asad J, Jacobson AF, Estabrook A, Smith SR, Boolbol SK, Feldman $\mathrm{SM}$, et al. Does oncotype DX recurrence score affect the management of patients with early-stage breast cancer? Am J Surg. (2008) 196:52729. doi: 10.1016/j.amjsurg.2008.06.021

35. Li S, Lang GT, Zhang YZ, Yu KD, Shao ZM, Zhang Q. Interaction between glutathione S-transferase M1-null/present polymorphism and adjuvant chemotherapy influences the survival of breast cancer. Cancer Med. (2018) 7:4202-7. doi: 10.1002/cam4.1567
36. Campos CZ, Losi Guembarovski R, de Oliveira CEC, Banin Hirata BK, Vitiello GAF, Dias FL, et al. Glutathione S-transferases deletions may act as prognosis and therapeutic markers in breast cancer. Clin Exp Med. (2018) 18:27-35. doi: 10.1007/s10238-017-0461-6

37. Pelekanou V, Villarroel-Espindola F, Schalper KA, Pusztai L, Rimm DL. CD68, CD163, and matrix metalloproteinase 9 (MMP-9) colocalization in breast tumor microenvironment predicts survival differently in ER-positive and -negative cancers. Breast Cancer Res. (2018) 20:154. doi: 10.1186/s13058-018-1076-x

38. Li W, Liang RR, Zhou C, Wu MY, Lian L, Yuan GF, et al. The association between expressions of Ras and CD68 in the angiogenesis of breast cancers. Cancer Cell Int. (2015) 15:17. doi: 10.1186/s12935-0150169-1

39. Cobleigh MA, Tabesh B, Bitterman P, Baker J, Cronin M, Liu ML, et al. Tumor gene expression and prognosis in breast cancer patients with 10 or more positive lymph nodes. Clin Cancer Res. (2005) 11:862331. doi: 10.1158/1078-0432.CCR-05-0735

40. Hao X, Sun B, Hu L, Lahdesmaki H, Dunmire V, Feng Y, et al. Differential gene and protein expression in primary breast malignancies and their lymph node metastases as revealed by combined cDNA microarray and tissue microarray analysis. Cancer. (2004) 100:1110-22. doi: 10.1002/cncr.20095

41. Kroigard AB, Larsen MJ, Thomassen M, Kruse TA. Molecular concordance between primary breast cancer and matched metastases. Breast J. (2016) 22:420-30. doi: 10.1111/tbj.12596

42. Boolbol SK, Harshan M, Chadha M, Kirstein L, Cohen JM, Klein P, et al. Genomic comparison of paired primary breast carcinomas and lymph node macrometastases using the oncotype DX breast recurrence score $(\mathrm{R})$ test. Breast Cancer Res Treat. (2019) 177:611-8. doi: 10.1007/s10549-019-05346-1

43. Thompson AM, Jordan LB, Quinlan P, Anderson E, Skene A, Dewar JA, et al Prospective comparison of switches in biomarker status between primary and recurrent breast cancer: the Breast Recurrence In Tissues Study (BRITS). Breast Cancer Res. (2010) 12:R92. doi: 10.1186/bcr2771

44. Amir E, Miller N, Geddie W, Freedman O, Kassam F, Simmons C, et al. Prospective study evaluating the impact of tissue confirmation of metastatic disease in patients with breast cancer. J Clin Oncol. (2012) 30:58792. doi: 10.1200/JCO.2010.33.5232

45. Bollet MA, Servant N, Neuvial P, Decraene C, Lebigot I, Meyniel JP, et al. High-resolution mapping of DNA breakpoints to define true recurrences among ipsilateral breast cancers. J Natl Cancer Inst. (2008) 100:4858. doi: $10.1093 /$ jnci/djm 266

Conflict of Interest: The authors declare that the research was conducted in the absence of any commercial or financial relationships that could be construed as a potential conflict of interest.

Copyright (C) 2020 Lu, Tong, Huang, Lin, Wu, Fei, Huang, He, Zhu, Chen, Li, Chen and Shen. This is an open-access article distributed under the terms of the Creative Commons Attribution License (CC BY). The use, distribution or reproduction in other forums is permitted, provided the original author(s) and the copyright owner(s) are credited and that the original publication in this journal is cited, in accordance with accepted academic practice. No use, distribution or reproduction is permitted which does not comply with these terms. 